\title{
CORRELATOR ANALYSIS OF MULTIPARTICLE EVENTS
}

\author{
N. Amelin, P. Filip, R. Lednicky, M. Pachr \\ Joint Institute for Nuclear Research, \\ Dubna, Moscow Region, 141980, Russia
}

\begin{abstract}
A procedure for the evaluation of correlators of any order in a reasonable computer time is presented. Connection between correlators and fluctuations of the event mean values of observables is discussed. Extension of the procedure to event-by-event approach is suggested. The usefulness of the method is demonstrated using the events simulated within various models of multipaticle production.
\end{abstract}

Keywords: particle correlations, correlators, mean value fluctuations

\section{Introduction}

Recent and near-future experiments devoted to the study of relativistic nuclear collisions (see, e.g., $[1,2]$ ) have stirred up considerable interest in suitable methods of the analysis of high particle multiplicity events. For example, fluctuations of various observables are widely studied using the event-byevent approach [3]. In this paper we consider the integral characteristics of particle correlations, namely the correlators [4] for a given particle observable (e.g., the particle energy $E$, transverse momentum $p_{t}$ or rapidity $y$ ). We suggest a fast procedure how to construct these quantities. We also investigate their properties, including the connection with the event-to-event fluctuations, and their ability to reflect underlying mechanisms in the case of several models.

The paper is now organized as follows. In section 2 we give the formal definition of correlators and discuss some of their properties. Sections 3-5 deal with the evaluation of correlators from experimental data. In section 4 we also consider the connection of the correlators with the fluctuations of the 
observable event-mean values. In section 6 we apply the proposed procedure to events simulated within various models of particle production. The results are summarized in section 6. In Appendix we derive the expressions allowing one to relate the correlators with the central moments of the single-particle distributions and the event-to-event fluctuations of the observable mean.

\section{Particle correlators}

Let us start with events of fixed multiplicity $n$ of the produced or observed particles of a given kind. The inclusive or exclusive production probability of $\nu \leq n$ identical particles can be described by a multivariate probability distribution function (PDF) $f_{\nu}^{(n)}\left(x_{1}, \ldots, x_{\nu}\right)$ of a given observable $x$, where $f_{\nu}^{(n)}$ is a symmetric function normalized to unity:

$$
\int f_{\nu}^{(n)}\left(x_{1}, \ldots, x_{\nu}\right) d x_{1} \ldots d x_{\nu}=1
$$

The PDF can be characterized by the mean value

$$
\bar{x}^{(n)}=\int x f_{1}^{(n)}(x) d x
$$

and - by the $l$-order moment-type quantities

$$
C_{i_{1}, \ldots, i_{l}}^{(n, x)}=\int\left(x_{i_{1}}-\bar{x}^{(n)}\right) \ldots\left(x_{i_{l}}-\bar{x}^{(n)}\right) f_{n}^{(n)}\left(x_{1}, \ldots, x_{n}\right) d x_{1} \ldots d x_{n} .
$$

Due to the PDF symmetry, all the quantities $C_{i_{1}, \ldots, i_{l}}^{(n, x)}$ coincide for any permutation of indexes.

In the case of independent production of particles of a given kind the multivariate PDF reduces to the product of the same one-particle distribution functions (neglecting the correlations due to quantum statistics and final state interaction):

$$
f_{\nu}^{(n)}\left(x_{1}, \ldots, x_{\nu}\right)=f_{1}^{(n)}\left(x_{1}\right) \ldots f_{1}^{(n)}\left(x_{\nu}\right)
$$

Obviously, in this case $C_{i_{1}, i_{2}, \ldots i_{l}}^{(n,)_{1}}=0$ for $i_{1} \neq i_{2} \ldots \neq i_{l}$. The quantities $C_{i_{1}, i_{2}, \ldots, i_{l}}^{(n, x)}$ for $i_{1} \neq i_{2} \ldots \neq i_{l}$ thus measure a correlation among produced particles and therefore we will call them correlators. Since correlators do not 
depend on the particular set of $l$ mutually different indexes, we will use for them the simple notation $C_{l}^{(n, x)}$. Note that for equal indexes $i_{1}=i_{2}=\ldots=$ $i_{l}, C_{i_{1}, i_{2}, \ldots i_{l}}^{(n, x)} \equiv M_{l}^{(n, x)}$ represents the $l$-th central moment of the one-particle PDF.

In practice, number $n$ of produced or observed particles of a given kind can vary from 0 to the ultimate multiplicity $n_{\max }$, so one has to introduce the corresponding probabilities $p_{n}$ and rewrite Eqs. (2) and (3) as

$$
\begin{gathered}
\langle\bar{x}\rangle \equiv\left\langle\bar{x}^{(n)}\right\rangle=\sum_{n=1}^{n_{\max }} p_{n} \bar{x}^{(n)} / \sum_{n=1}^{n_{\max }} p_{n}=\sum_{n=1}^{n_{\max }} p_{n} \int x f_{1}^{(n)}(x) d x / \sum_{n=1}^{n_{\max }} p_{n}, \\
C_{l}^{(x)}=\sum_{n=l}^{n_{\max }} p_{n} . \\
\cdot \int\left(x_{1}-\langle\bar{x}\rangle\right) \ldots\left(x_{l}-\langle\bar{x}\rangle\right) f_{l}^{(n)}\left(x_{1}, \ldots, x_{l}\right) d x_{1} \ldots d x_{l} / \sum_{n=l}^{n_{\max }} p_{n} .
\end{gathered}
$$

Generally, the PDF can depend on various event characteristics $\alpha$, including the observed multiplicity, the particle composition or the selected range of the impact parameters. Eqs. (5) and (6) should be then generalized by the substitution $\sum p_{n} \rightarrow \sum p_{\alpha}$. Particularly,

$$
\langle\bar{x}\rangle=\left\langle\bar{x}^{(n)}\right\rangle=\sum_{\alpha} p_{\alpha} \bar{x}^{(\alpha)} / \sum_{\alpha} p_{\alpha}=\sum_{\alpha} p_{\alpha} \int x f_{1}^{(\alpha)}(x) d x / \sum_{\alpha} p_{\alpha}
$$

Using the identity

$$
x_{k}-\langle\bar{x}\rangle=\left(x_{k}-\bar{x}^{(\alpha)}\right)+\left(\bar{x}^{(\alpha)}-\langle\bar{x}\rangle\right),
$$

one can rewrite the global correlator $C_{l}^{(x)}$ in terms of the $\alpha$-dependent correlators

$$
C_{l}^{(\alpha, x)}=\int\left(x_{1}-\left\langle\bar{x}^{(\alpha)}\right\rangle\right) \ldots\left(x_{l}-\left\langle\bar{x}^{(\alpha)}\right\rangle\right) f_{l}^{(\alpha)}\left(x_{1}, \ldots, x_{l}\right) d x_{1} \ldots d x_{l}
$$

and the fluctuations of the observable mean $\bar{x}^{(\alpha)}$ at a given $\alpha$ around the global mean $\langle\bar{x}\rangle$ :

$$
C_{l}^{(x)}=\sum_{\alpha} p_{\alpha} \sum_{\lambda=0}^{l}\left(\begin{array}{l}
l \\
\lambda
\end{array}\right) C_{\lambda}^{(\alpha, x)}\left(\bar{x}^{(\alpha)}-\langle\bar{x}\rangle\right)^{l-\lambda} / \sum_{\alpha} p_{\alpha}
$$




$$
\equiv\left\langle\sum_{\lambda=0}^{l}\left(\begin{array}{l}
l \\
\lambda
\end{array}\right) C_{\lambda}^{(\alpha, x)}\left(\bar{x}^{(\alpha)}-\langle\bar{x}\rangle\right)^{l-\lambda}\right\rangle,
$$

where $C_{0}^{(\alpha, x)}=1, C_{1}^{(\alpha, x)}=0$. One may see that the absence of the correlation at any $\alpha$ (i.e. $C_{l}^{(\alpha, x)}=0$ for $l>1$ ) does not lead to a vanishing global correlator. In this case, the latter is solely determined by the fluctuation of the $\alpha$-dependent observable mean: $C_{l}^{(x)}=\left\langle\left(\bar{x}^{(\alpha)}-\langle\bar{x}\rangle\right)^{l}\right\rangle$.

The above formalism ignores the possible non-identity of the selected particles. Its generalization to the correlators of different particle species is however straightforward. For example, for two types of particles, say those characterized by positive $(+)$ and negative $(-)$ charge, one has to make the substitutions $l \rightarrow l_{+}, l_{-}, x \rightarrow x_{+}$and $x_{-}$. Particularly,

$$
\begin{gathered}
\bar{x}_{+}^{(\alpha)}=\int x_{+} f_{1,0}^{(\alpha)}\left(x_{+}\right) d x_{+}, \quad \bar{x}_{-}^{(\alpha)}=\int x_{-} f_{0,1}^{(\alpha)}\left(x_{-}\right) d x_{-}, \\
C_{l_{+}, l_{-}}^{(\alpha, x)}=\int\left(x_{+_{1}}-\left\langle\bar{x}_{+}^{(\alpha)}\right\rangle\right) \ldots\left(x_{+_{l_{+}}}-\left\langle\bar{x}_{+}^{(\alpha)}\right\rangle\right)\left(x_{-_{1}}-\left\langle\bar{x}_{-}^{(\alpha)}\right\rangle\right) \ldots\left(x_{-_{-}}-\left\langle\bar{x}_{-}^{(\alpha)}\right\rangle\right) . \\
f_{l_{+}, l_{-}}^{(\alpha)}\left(x_{+_{1}}, \ldots, x_{+_{l_{+}}} ; x_{-_{1}}, \ldots, x_{-_{l_{-}}}\right) d x_{+_{1}} \ldots d x_{+_{l_{+}}} d x_{-_{1}} \ldots d x_{-_{l_{-}}}
\end{gathered}
$$

It is instructive to express the correlator $C_{2}^{(x)} \equiv C_{c c}^{(x)}$ of two charged particles in the events with $n=n_{+}+n_{-}$selected particles through the correlators $C_{2,0}^{(x)} \equiv C_{++}^{(x)}, C_{0,2}^{(x)} \equiv C_{--}^{(x)}$ and $C_{1,1}^{(x)} \equiv C_{+-}^{(x)}$. Using the identity

$$
\bar{x}=\bar{x}_{ \pm}-\left(\bar{x}_{ \pm}-\bar{x}\right) \equiv \bar{x}_{ \pm}-\Delta \bar{x}_{ \pm},
$$

one can write

$$
C_{c c}^{(x)}=\frac{n_{++}}{n_{c c}}\left[C_{++}^{(x)}+\Delta \bar{x}_{+}^{2}\right]+\frac{n_{--}}{n_{c c}}\left[C_{--}^{(x)}+\Delta \bar{x}_{-}^{2}\right]+\frac{n_{+-}}{n_{c c}}\left[C_{+-}^{(x)}+\Delta \bar{x}_{+} \Delta \bar{x}_{-}\right],
$$

where

$$
n_{c c}=n_{++}+n_{--}+n_{+-}=n_{+}\left(n_{+}-1\right) / 2+n_{-}\left(n_{-}-1\right) / 2+n_{+} n_{-}
$$

is the number of charged pairs. One may see that even in the absence of correlations of particles of given charges, i.e. $C_{++}^{(x)}=C_{--}^{(x)}=C_{+-}^{(x)}=0$, the correlator $C_{c c}^{(x)}$ can be non-zero provided $\bar{x}_{+} \neq \bar{x}_{-}$. In particular, for $n_{+}=n_{-}=n / 2$,

$$
\bar{x}=\frac{n_{+}}{n} \bar{x}_{+}+\frac{n_{-}}{n} \bar{x}_{-}=\frac{1}{2}\left(\bar{x}_{+}+\bar{x}_{-}\right), \quad \Delta \bar{x}_{+}=-\Delta \bar{x}_{-}=\frac{1}{2}\left(\bar{x}_{+}-\bar{x}_{-}\right)
$$


and, in the absence of correlations,

$$
C_{c c}^{(x)}=-\frac{\left(\bar{x}_{+}-\bar{x}_{-}\right)^{2}}{4(n-1)} \leq 0
$$

\section{Correlator estimates}

Let us first consider $n_{\text {evt }}$ experimental events with a fixed multiplicity $n$. The mean value of the observable $x$ can then be estimated as

$$
\langle\bar{x}\rangle=\frac{1}{n_{\mathrm{evt}}} \sum_{i=1}^{n_{\mathrm{evt}}} \bar{x}^{(i)},
$$

where $\bar{x}^{(i)}$ is the estimate of the observable mean in the $i$-th event:

$$
\bar{x}^{(i)}=\frac{1}{n} \sum_{j=1}^{n} x_{j}^{(i)} .
$$

Similarly, the correlator $C_{l}^{(x)}$ can be estimated as

$$
C_{l}^{(x)}=\frac{1}{n_{\mathrm{evt}}} \sum_{i=1}^{n_{\mathrm{evt}}} C_{l}^{(i, x)}
$$

where $C_{l}^{(i, x)}$ is the estimate of the correlator in the $i$-th event:

$$
C_{l}^{(i, x)}=\frac{1}{n_{l}} \sum\left(x_{i_{1}}^{(i)}-\langle\bar{x}\rangle\right) \ldots\left(x_{i_{l}}^{(i)}-\langle\bar{x}\rangle\right)
$$

the sum in Eq. (21) runs over all $n_{l}$ sets of $l$ particles chosen from $n$ particles in the event. One can sum either over $n_{l}=\left(\begin{array}{l}n \\ l\end{array}\right)=n(n-1) \ldots(n-l+1) / l$ ! of the sorted sets $i_{1}<i_{2}<\ldots<i_{l}$ or over $n_{l}=n(n-1) \ldots(n-l+1)$ of the unsorted sets $i_{1} \neq i_{2} \neq \ldots \neq i_{l}$. In the latter case, each of the unsorted sets gives rise to the $l$ ! of identical terms in the sum.

In the case of a mixture of events with different multiplicities $n^{(i)}$, one can estimate the observable mean and the correlators in a similar way as in the two-step averaging procedure given in Eqs. (18)-(21). One should only make substitutions $n \rightarrow n^{(i)}, n_{l} \rightarrow n_{l}^{(i)}$ in Eqs. (19), (21) and take into account 
that the single-event averages enter into Eqs. (18) and (20) multiplied by the weights proportional to $n^{(i)}$ and $n_{l}^{(i)}$, respectively:

$$
\begin{gathered}
\langle\bar{x}\rangle \equiv\left\langle\bar{x}^{(i)}\right\rangle=\frac{1}{N} \sum_{i=1}^{n_{\mathrm{evt}}} n^{(i)} \bar{x}^{(i)}, \quad N=\sum_{i=1}^{n_{\mathrm{evt}}} n^{(i)}, \\
C_{l}^{(x)} \equiv\left\langle C_{l}^{(i, x)}\right\rangle_{l}=\frac{1}{N_{l}} \sum_{i=1}^{n_{\mathrm{evt}}} n_{l}^{(i)} C_{l}^{(i, x)}, \quad N_{l}=\sum_{i=1}^{n_{\mathrm{evt}}} n_{l}^{(i)} .
\end{gathered}
$$

The same result can be obtained by averaging simply over all $N$ collected particles or all $N_{l} l$-particle sets (formed from particles within the same event only):

$$
\begin{gathered}
\langle\bar{x}\rangle=\frac{1}{N} \sum_{j=1}^{N} x_{j} \\
C_{l}^{(x)}=\frac{1}{N_{l}} \sum\left(x_{i_{1}}-\langle\bar{x}\rangle\right) \ldots\left(x_{i_{l}}-\langle\bar{x}\rangle\right),
\end{gathered}
$$

where the sum in Eq. (25) runs over all $N_{l}$ sets.

The generalization of Eqs. (18)-(25) to two or more particle species is straightforward. For example, for two types of particles characterized by positive and negative charge, one has to make substitutions $l \rightarrow l_{+}, l_{-}, n \rightarrow$ $n_{+}, n_{-}, n_{l} \rightarrow n_{l_{+}}, n_{l-}, N_{l} \rightarrow \sum_{i} n_{l_{+}}^{(i)} n_{l-}^{(i)}$ and $x \rightarrow x_{+}, x_{-}$. Particularly,

$$
C_{1,1}^{(i, x)}=\left(\bar{x}_{+}^{(i)}-\left\langle x_{+}\right\rangle\right)\left(\bar{x}_{-}^{(i)}-\left\langle x_{-}\right\rangle\right), \quad C_{l_{+}, l_{-}}^{(i, x)}=C_{l_{+}}^{\left(i, x_{+}\right)} C_{l_{-}}^{\left(i, x_{-}\right)} .
$$

To calculate the errors, we can split all the events into $n_{g}$ subgroups, each with about the same number of events, and estimate the correlator $C^{(m)}$ (we omit all other indexes for the sake of simplicity) in the $m$-th subgroup using the global estimate of the mean value $\langle x\rangle$. The global estimate of the correlator is then given by the mean of the group values:

$$
C=\frac{1}{n_{g}} \sum_{m=1}^{n_{g}} C^{(m)} .
$$

The dispersion of the group values is

$$
D=\frac{1}{n_{g}-1} \sum_{m=1}^{n_{g}}\left(C^{(m)}-C\right)^{2},
$$


where the sum of the deviations squared is divided by $n_{g}-1$ since one degree of freedom is used to determine the global estimate of the correlator according to Eq. (27). Neglecting a small correlation of the group correlators due to the use of the global estimate of $\langle x\rangle$, one can calculate the error in the global correlator estimate as

$$
\sigma(C)=\left(D / n_{g}\right)^{1 / 2}=\left(\frac{1}{n_{g}\left(n_{g}-1\right)} \sum_{m=1}^{n_{g}}\left(C^{(m)}-C\right)^{2}\right)^{1 / 2} .
$$

\section{Correlators and event-to-event fluctuations}

To relate the correlator $C_{l}^{(x)}$ with the event-to-event fluctuation of the observable single-event mean $\bar{x}^{(i)}$, one can use the analog of the identity in Eq. (8) with the substitutions $x_{k} \rightarrow x_{k}^{(i)}$ and $\bar{x}^{(\alpha)} \rightarrow \bar{x}^{(i)}$, and rewrite the estimate of the single-event correlator in Eq. (21) in the form

$$
C_{l}^{(i, x)}=\sum_{\lambda=0}^{l}\left(\begin{array}{l}
l \\
\lambda
\end{array}\right) c_{\lambda}^{(i, x)}\left(\bar{x}^{(i)}-\langle\bar{x}\rangle\right)^{l-\lambda},
$$

where $c_{0}^{(i, x)}=1, c_{1}^{(i, x)}=0$ and $c_{l}^{(i, x)}$ for $l \geq 2$ is defined similar to Eq. (21) except for the substitution $\langle\bar{x}\rangle \rightarrow \bar{x}^{(i)}$ :

$$
c_{l}^{(i, x)}=\frac{1}{n_{l}^{(i)}} \sum\left(x_{i_{1}}^{(i)}-\bar{x}^{(i)}\right) \ldots\left(x_{i_{l}}^{(i)}-\bar{x}^{(i)}\right) .
$$

The estimate of the correlator can then be written in the form

$$
C_{l}^{(x)}=\left\langle\sum_{\lambda=0}^{l}\left(\begin{array}{l}
l \\
\lambda
\end{array}\right) c_{\lambda}^{(i, x)}\left(\bar{x}^{(i)}-\langle\bar{x}\rangle\right)^{l-\lambda}\right\rangle_{l}
$$

where the $l$-dependent averaging is defined in Eq. (23). Introducing the notation

$$
\Delta \bar{x}^{(i)}=\bar{x}^{(i)}-\langle\bar{x}\rangle
$$

and omitting the event indexes, Eq. (32) particularly yields:

$$
\begin{gathered}
C_{2}^{(x)}=\left\langle c_{2}^{(x)}+\Delta \bar{x}^{2}\right\rangle_{2}, \\
C_{3}^{(x)}=\left\langle c_{3}^{(x)}+3 c_{2}^{(x)} \Delta \bar{x}+\Delta \bar{x}^{3}\right\rangle_{3},
\end{gathered}
$$




$$
C_{4}^{(x)}=\left\langle c_{4}^{(x)}+4 c_{3}^{(x)} \Delta \bar{x}+6 c_{2}^{(x)} \Delta \bar{x}^{2}+\Delta \bar{x}^{4}\right\rangle_{4} .
$$

For two different particle species + and - , Eqs. (26) and (32) yield, e.g.:

$$
C_{1,1}^{(x)} \equiv C_{+-}^{(x)}=\left\langle\Delta \bar{x}_{+} \Delta \bar{x}_{-}\right\rangle_{1,1}, \quad C_{2,1}^{(x)} \equiv C_{++-}^{(x)}=\left\langle\left(c_{2}^{(x+)}+\Delta \bar{x}_{+}{ }^{2}\right) \Delta \bar{x}_{-}\right\rangle_{2,1} .
$$

The meaning of quantities $c_{l}^{(x)}$ is clarified in Appendix, where it is shown that they can be expressed through the estimates of the moments of the single-particle $x$-distribution in a given event:

$$
m_{\lambda}^{(x)}=\frac{1}{n} \sum_{j=1}^{n}\left(x_{j}-\bar{x}\right)^{\lambda} .
$$

Particularly,

$$
\begin{gathered}
c_{2}^{(x)}=-\frac{m_{2}^{(x)}}{n-1}, \\
c_{3}^{(x)}=\frac{2 m_{3}^{(x)}}{(n-1)(n-2)}, \\
c_{4}^{(x)}=\frac{3 n\left(m_{2}^{(x)}\right)^{2}-6 m_{4}^{(x)}}{(n-1)(n-2)(n-3)},
\end{gathered}
$$

One may conclude from Eqs. (34)-(37) and Eqs. (39)-(41):

- Quantities $c_{l}^{(x)}$ are determined by the shape of single-particle $x$-distribution and by multiplicity of detected $(n)$ or selected $(n \rightarrow \nu \leq n)$ particles in a given event. Therefore, they are sensitive only to a part of the correlation related to this shape; an example is the correlation due to energy-momentum conservation (see the 4-th item). The remaining part is contained in the event-to-event fluctuations of the observable mean in accordance with Eqs. (32)-(37).

- The magnitude of the quantities $c_{l}^{(\nu, x)}$ decreases with the increasing number $\nu$ of selected particles. This decrease should be compensated by the $\nu$-dependence of the fluctuations of single-event mean observable $\bar{x}^{(i)}$ to guarantee the $\nu$-independence of the correlators. Particularly, for uncorrelated production $\left(C_{l}^{(x)}=0\right)$, one gets $\left\langle\Delta \bar{x}^{2}\right\rangle_{2}^{(\nu)}=\left\langle m_{2}^{(x)}\right\rangle_{2} /(\nu-$ $1)$. 
- Assuming the moments $m_{l}^{(x)}$ of the single-particle distribution weakly varying with the number $n$ of observed particles, the correlators for high enough $n$ are dominated by the event-to-event fluctuations, i.e. $C_{l}^{(x)} \rightarrow\left\langle\Delta \bar{x}^{l}\right\rangle_{l}$.

- For a conserved additive observable $x$ (e.g., particle energy), considering particles of any kind and assuming that all $n_{\text {tot }}$ particles are observed $\left(n=n_{\text {tot }}\right)$, the mean $\bar{x}$ does not fluctuate so $C_{l}^{(x)}=\left\langle c_{l}^{(x)}\right\rangle_{l}$. Particularly, $C_{2}^{(x)}=-\left\langle m_{2}^{(x)}\right\rangle_{2} /\left(n_{\text {tot }}-1\right)$ and $C_{3}^{(x)}=2\left\langle m_{3}^{(x)}\right\rangle_{3} /\left[\left(n_{\text {tot }}-\right.\right.$ $\left.1)\left(n_{\text {tot }}-2\right)\right]$ for the events with about the same numbers of produced particles.

- Evaluation of correlators $C_{l}^{(x)}$ using Eqs. (39)-(41) can save substantial amount of computing time compared with the direct evaluation according to Eq. (25). In the former case the number of operations is $\propto n$ while in the latter case it is $\propto n^{l}$.

\section{Event-by-event correlators}

In the case of uncorrelated particle production, the event-to-event fluctuations of the observable mean are solely determined by the quantities $c_{l}^{(x)}$. Such "standard" fluctuations are thus related to the single-particle distributions and can be estimated by the event-mixing techniques. The eventual deviation from the "standard" or "statistical" fluctuations then signals the presence of correlations or - a mixture of the events with different singleparticle distributions.

Therefore, to clarify the origin of the "non-standard" or "non-statistical" fluctuations, it is desirable to estimate the correlators for the events with similar characteristics $\alpha$. The ultimate solution is to use the information from a given event only. To do so, one has to destroy the equality in Eq. (A.1), i.e. decouple as much as possible the observables $x_{j}$ entering into the correlator estimate from the observable mean $\bar{x}$ in a given event. In the case of sufficiently large multiplicity, it can be achieved by splitting the event in a number of sub-events $s=1,2, \ldots, n_{\text {sevt }}$ with about the same multiplicities $n^{(s)}$. One can then estimate the correlators according to Eqs. (18)-(25) or (34)-(37) and (38)-(41), making the substitutions $i \rightarrow s$ and $n_{\text {evt }} \rightarrow n_{\text {sevt }}$. 
Table 1: The correlators corresponding to PDF's in Eqs. (42) (the first two lines) and (43). The results of numerical integration are compared with the estimates obtained from $n_{\text {evt }}$ simulated events. The allowed interval of the variable $x$ was $0-150$, the parameters $T=150, T_{1}=25$ and $A=0,1,2$.

\begin{tabular}{|c|c|c|c|}
\hline Correlator & $C_{2}^{(x)}$ & $C_{3}^{(x)}$ & $C_{4}^{(x)}$ \\
\hline$n=3$ & 1312.5 & 0. & \\
$n_{\mathrm{evt}}=5 \cdot 10^{5}$ & $1313.4 \pm 1.8$ & $61 . \pm 108$. & \\
\hline$n=3, A=0$ & 0. & 0. & \\
$n_{\mathrm{evt}}=5 \cdot 10^{5}$ & $-0.3 \pm 1.4$ & $21 . \pm 70$. & \\
\hline$n=3, A=2$ & 229.0 & 1771.5 & \\
$n_{\mathrm{evt}}=5 \cdot 10^{5}$ & $229.9 \pm 1.4$ & $1779 . \pm 110$. & \\
\hline $\mathrm{n}=4, \mathrm{~A}=1$ & 113.8 & 707. & 2913. \\
$n_{\mathrm{evt}}=3 \cdot 10^{6}$ & $114.4 \pm 0.5$ & $666 . \pm 23$. & $3890 . \pm 1836$. \\
\hline
\end{tabular}

\section{$6 \quad$ Examples}

Let us first consider the following simple PDF's characterized by the parameters $T, T_{1}$ and $A$ :

$$
\begin{aligned}
f_{3}^{(3)}\left(x_{1}, x_{2}, x_{3}\right) & \left.\propto e^{-\left|\frac{x_{1}-x_{2}}{T_{1}}\right|} e^{-\left|\frac{x_{1}-x_{3}}{T_{1}}\right|} e^{-\left|\frac{x_{2}-x_{3}}{T_{1}}\right|}\right), \\
f_{n}^{(n)}\left(x_{1}, x_{2}, \ldots, x_{n}\right) & \propto \prod_{i=1}^{n} e^{-\frac{x_{i}}{T}}\left(1+A \sum_{i<j \leq n} e^{-\left|\frac{x_{i}-x_{j}}{T_{1}}\right|}\right),
\end{aligned}
$$

The parameter $A$ in Eq. (43) controls the correlation strength; for $A=0$ there are no correlations among particles, so $C_{l}^{(x)}=0$. Eq. (42) corresponds to two-particle correlations only, thus leading to $C_{3}^{(x)}=0$.

The correlator expectation values calculated according to PDF's in Eqs. (42) and (43) by direct numerical integration and their estimates obtained from simulated events are compared in table 1 . One may see that the estimated values of the correlators agree with their expectation values within the errors. The relative errors rapidly increase with the order $l$ of the correlator thus making quite uneasy its measurement for $l>4$. 
Table 2: Correlators $C_{2}^{(x)}$ and $C_{3}^{(x)}$ estimated from the "microcanonical" ensemble of events with fixed mean particle energy $\bar{x}=100$ in arbitrary units, each consisting of $2 \cdot 10^{4}$ events with fixed particle multiplicity $n_{\text {tot }}$. All particles are assumed to be observed, i.e. $n=n_{\text {tot }}$.

\begin{tabular}{|l|c|c|c|c|c|}
\hline$n_{\text {tot }}$ & 5 & 10 & 15 & 20 & 100 \\
\hline$C_{2}^{(x)}$ & $-1181 \pm 2$ & $-624 \pm 1$ & $-426 \pm 1$ & $-323 \pm 1$ & $-66.2 \pm 0.1$ \\
\hline$C_{3}^{(x)}$ & $49900 \pm 200$ & $14730 \pm 50$ & $6940 \pm 20$ & $4041 \pm 6$ & $174 \pm 1$ \\
\hline
\end{tabular}

Table 3: The same as in table 2 for $n_{\text {tot }}=100$ and different numbers $\nu$ of selected particles.

\begin{tabular}{|c|c|c|c|c|}
\hline$\nu$ & 5 & 10 & 15 & 20 \\
\hline$C_{2}^{(x)}$ & $-75 \pm 8$ & $-63 \pm 3$ & $-68 \pm 2$ & $-66 \pm 2$ \\
\hline$C_{3}^{(x)}$ & $200 \pm 500$ & $300 \pm 100$ & $220 \pm 70$ & $170 \pm 50$ \\
\hline
\end{tabular}

As another example, we consider the energy correlators estimated from the "microcanonical" ensemble of events simulated according to the nonrelativistic phase space (non-relativistic ideal gas of particles) using Metropolis algorithm to redistribute the particle energies via binary energy-conserving collisions. The total amount of energy distributed to $n_{\text {tot }}$ particles is $X_{\text {tot }}=$ $n_{\text {tot }} \bar{x}$, where $\bar{x}$ is the mean particle energy. We have put $\bar{x}=100$ in arbitrary units. Assuming that all $n_{\text {tot }}$ particles are observed, the mean particle energy does not fluctuate $(\Delta \bar{x}=0)$ and the correlators $C_{l}^{(x)}$ are then given by Eqs. (39)-(41) with $n=n_{\text {tot. }}$. Particularly, $C_{2}^{(x)}$ and $C_{3}^{(x)}$ vanish at large $n_{\text {tot }}$ as $1 / n_{\text {tot }}$ and $1 / n_{\text {tot }}{ }^{2}$ respectively. The results shown in table 2 confirm this behavior.

In table 3 , we show the same correlators as in table 2 for $n_{\text {tot }}=100$, but now calculated for different numbers of selected particles $\nu<n_{\text {tot }}$. One may see that within the errors the correlator estimates are $\nu$-independent. This confirms the conclusion at the end of section 4 about the compensation of 
Table 4: The estimates of the pion transverse momentum correlators obtained from $2 \cdot 10^{3}$ simulated central PSM events of $\mathrm{Pb}+\mathrm{Pb}$ collisions at total c.m. energy $\sqrt{s}=200 \mathrm{AGeV}$.

\begin{tabular}{|c|c|c|c|}
\hline Species & $\begin{array}{c}C_{2}^{\left(p_{t}\right)} \\
(\mathrm{GeV} / \mathrm{c})^{2}\end{array}$ & $\begin{array}{c}C_{3}^{\left(p_{t}\right)} \\
(\mathrm{GeV} / \mathrm{c})^{3}\end{array}$ & $\begin{array}{c}C_{4}^{\left(p_{t}\right)} \\
(\mathrm{GeV} / \mathrm{c})^{4}\end{array}$ \\
\hline$\pi^{+}$ & $(44.0 \pm 6.6) \cdot 10^{-6}$ & $(-7.4 \pm 12.1) \cdot 10^{-8}$ & $(8.7 \pm 3.3) \cdot 10^{-9}$ \\
\hline$\pi^{-}$ & $(44.6 \pm 6.0) \cdot 10^{-6}$ & $(7.8 \pm 12.5) \cdot 10^{-8}$ & $(9.5 \pm 4.6) \cdot 10^{-9}$ \\
\hline$\pi^{0}$ & $(71.2 \pm 7.6) \cdot 10^{-6}$ & $(3.0 \pm 2.7) \cdot 10^{-7}$ & $(34.3 \pm 9.5) \cdot 10^{-9}$ \\
\hline$\pi^{ \pm}$ & $(52.6 \pm 4.2) \cdot 10^{-6}$ & $(-1.5 \pm 7.1) \cdot 10^{-8}$ & $(9.0 \pm 2.0) \cdot 10^{-9}$ \\
\hline$\pi^{ \pm, 0}$ & $(59.6 \pm 3.5) \cdot 10^{-6}$ & $(2.1 \pm 5.3) \cdot 10^{-8}$ & $(11.2 \pm 1.6) \cdot 10^{-9}$ \\
\hline
\end{tabular}

the $\nu$-dependence of the quantities $c_{l}^{(\nu, x)}$ by that of the $\bar{x}$ fluctuations.

Finally, we have estimated the pion transverse momentum correlators $C_{2}^{\left(p_{t}\right)}, C_{3}^{\left(p_{t}\right)}$ and $C_{4}^{\left(p_{t}\right)}$ using $2 \cdot 10^{3}$ central (impact parameter $b=0 \mathrm{fm}$ ) events of $\mathrm{Pb}+\mathrm{Pb}$ collisions at total c.m. energy $\sqrt{s}=200 \mathrm{AGeV}$ simulated in the parton string model (PSM) [5]. In this phenomenological model, the soft and semihard parton collisions initiate the formation of color strings and the subsequent string breaking leads to the production of stable (with respect to strong interaction) hadrons and resonances that are forced to decay. We have divided the simulated events into 100 subgroups and selected $\nu=1900$ pions of a given charge in each event. As one can see from table 4 , the correlators $C_{3}^{\left(p_{t}\right)}$ are consistent with zero within the errors while this is not the case for the correlators $C_{2}^{\left(p_{t}\right)}$ and $C_{4}^{\left(p_{t}\right)}$. The latter appear to be non-zero for any combination of pion charges, being somewhat higher for neutral pions. We have also selected $\pi^{+} \pi^{-}$pairs only and estimated $C_{+-}^{\left(p_{t}\right)}=(61.9 \pm 4.4) \cdot 10^{-6}(\mathrm{GeV} / \mathrm{c})^{2}$. Since the PSM takes into account only the resonances from the lowest $\mathrm{SU}(3)$ multiplets that do not decay into pairs of like-sign charged pions, the small difference between the correlators for pairs of like-sign and unlike-sign charged pions indicates that the non-zero PSM correlators are mainly of the non-resonance origin. Also, since we consider the high multiplicity events, the correlations due to energy-momentum conservation are of minor importance in accordance with the discussion in 
section 4. The probable source of the non-zero PSM correlators are thus the semihard parton collisions that are becoming important with the increasing energy and are known to lead to a noticeable "non-statistical" $\bar{p}_{t}$ fluctuation in $A u+A u$ collisions at RHIC [2].

\section{Conclusion}

We have developed a fast procedure allowing one to calculate, in a reasonable computer time, the particle correlators of any order and estimate their errors. The corresponding $\mathrm{C}++$ code is available on the request at e-mail address amelin@sunhe.jinr.ru. We have suggested the extension of this procedure for the event-by-event approach as well. We have shown a close relation between the correlators and fluctuations of the observable event-mean values. We have applied the proposed procedure to the events simulated within various models and demonstrated the usefulness of the two-, three- and four-particle correlators; the measurement of the higher-order correlators is rather difficult as it requires very high statistics.

\section{Acknowledgements}

We are grateful to J. Manjavidze and S. Shimanskii for useful discussions and - to A. Sissakian and Yu. Panebratsev for drawing our attention to this topic. This work was supported by the Grant Agency of the Czech Republic under contract 202/04/0793 and Slovak Grant Agency for Sciences under contract $2 / 4099 / 24$.

\section{Appendix A}

To clarify the meaning of quantities $c_{l}^{(x)}$, let us follow Ref. [6] and consider the powers of the equality

$$
0=\sum_{j=1}^{n}\left(x_{j}-\bar{x}\right) \equiv \sum_{j=1}^{n} \Delta_{j} .
$$


Thus, the second, third and fourth powers of Eq. (A.1) yield

$$
\begin{gathered}
0=\sum_{j \neq k} \Delta_{j} \Delta_{k}+\sum_{j} \Delta_{j}{ }^{2}, \\
0=\sum_{j \neq k \neq l} \Delta_{j} \Delta_{k} \Delta_{l}+\left(\begin{array}{l}
3 \\
2
\end{array}\right) \sum_{j \neq k} \Delta_{j} \Delta_{k}{ }^{2}+\sum_{j} \Delta_{j}{ }^{3}, \\
0=\sum_{j \neq k \neq l \neq m} \Delta_{j} \Delta_{k} \Delta_{l} \Delta_{m}+\left(\begin{array}{l}
4 \\
2
\end{array}\right) \sum_{j \neq k \neq l} \Delta_{j} \Delta_{k} \Delta_{l}{ }^{2}+ \\
\frac{1}{2 !}\left(\begin{array}{l}
4 \\
2
\end{array}\right)\left(\begin{array}{c}
4-2 \\
2
\end{array}\right) \sum_{j \neq k} \Delta_{j}{ }^{2} \Delta_{k}{ }^{2}+\left(\begin{array}{l}
4 \\
3
\end{array}\right) \sum_{j \neq k} \Delta_{j} \Delta_{k}{ }^{3}+\sum_{j} \Delta_{j}{ }^{4} .
\end{gathered}
$$

Using further the relations

$$
\begin{gathered}
\sum_{j \neq k} \Delta_{j} \Delta_{k}{ }^{\lambda}=-\sum_{k} \Delta_{k}{ }^{\lambda+1}, \\
\sum_{j \neq k \neq l} \Delta_{j} \Delta_{k} \Delta_{l}^{2}=\left(\sum_{j \neq k} \Delta_{j} \Delta_{k}\right) \sum_{l} \Delta_{l}{ }^{2}-2 \sum_{j \neq k} \Delta_{j} \Delta_{k}{ }^{3} \\
=-\left(\sum_{l} \Delta_{l}{ }^{2}\right)^{2}+2 \sum_{l} \Delta_{l}^{4},
\end{gathered}
$$

and

$$
\sum_{j \neq k} \Delta_{j}^{2} \Delta_{k}^{2}=\left(\sum_{l} \Delta_{l}^{2}\right)^{2}-\sum_{l} \Delta_{l}^{4}
$$

one can express the multiple sums in Eq. (31) through the sums of the powers of $\Delta_{j}$ related to the estimates of the central moments $m_{\lambda}^{(x)}$ in Eq. (38). Rewriting Eq. (31) in the form

$$
c_{l}^{(i, x)}=\frac{1}{n(n-1) \ldots(n-l+1)} \sum_{i_{1} \neq i_{2} \neq \ldots \neq i_{l}} \Delta_{i_{1}} \Delta_{i_{2}} \ldots \Delta_{i_{l}},
$$

one then proves Eqs. (39)-(41) relating quantities $c_{l}^{(x)}$ with central moments $m_{\lambda}^{(x)}$. 


\section{References}

[1] C. Pruneau, S. Gavin and S. Voloshin, Phys. Rev. C 66 (2002) 044904.

[2] J. Adams et al. (STAR), nucl-ex/0308033.

[3] Quark Matter 2004 Conference Proceedings, J.Phys. G30 (2004) Suppl.

[4] J. Manjavidze and A. Sissakian, Phys. Rep. 346 (2001) 1.

[5] N. Amelin, N. Armesto, C. Pajares and D. Sousa, Eur. Phys. J. C22 (2001) 149.

[6] A.V. Stadnik, N.I. Chernov and S.S. Shimanskii, JINR Report P112003-143, 2003. 